\title{
Extracranial head and neck schwannomas: a study of the nerve of origin
}

\author{
Hin-Lun Liu • Suet-Ying Yu • George Kam-Hop Li • \\ William Ignace Wei
}

Received: 27 October 2010/Accepted: 7 January 2011/Published online: 19 January 2011

(C) The Author(s) 2011. This article is published with open access at Springerlink.com

\begin{abstract}
Schwannoma is a type of benign nerve sheath tumour arising from the Schwann cell. Because of the close relationship between the tumour and the nerve of origin (NOO), the operation of extracranial head and neck schwannoma may lead to palsy of major nerve. For this reason, an accurate diagnosis of schwannoma with the identification of the NOO is crucial to the management. The aim of this review was to find out the distribution of the NOO and the usefulness of the investigations in the diagnosis of schwannoma. Medical records of the patients who underwent operation of the extracranial head and neck schwannoma in our division were reviewed. Between January 2000 and December 2009, 30 cases of extracranial head and neck schwannoma were operated. Sympathetic trunk $(10,33 \%)$ and vagus nerve $(6,20 \%)$ were the two most common NOOs. In five $(17 \%)$ cases, the NOO was not found to be arising from any major nerve. For these 30 patients, 20 received fine needle aspiration cytology (FNAC) and 26 underwent imaging studies (computed tomography or magnetic resonance imaging) before operation. The specificity of FNAC and imaging studies in making the diagnosis of schwannoma was 20 and $38 \%$, respectively. For the patients who had nerve palsies on presentation, their deficits remained after operation. The rate of nerve palsy after tumour excision with division of NOO and intracapsular enucleation was 100 and $67 \%$, respectively. The diagnosis of schwannoma is suggested by clinical features and supported by investigations. Most of the time, the diagnosis can only be confirmed on the
\end{abstract}

H.-L. Liu ( $\square)$ · S.-Y. Yu · G. K.-H. Li · W. I. Wei Division of Head and Neck Surgery, Department of Surgery, Queen Mary Hospital, University of Hong Kong Medical Centre, Pokfulam, Hong Kong, SAR, China

e-mail: lawrencehlliu@gmail.com histological study of the surgical specimen. Sympathetic trunk and vagus nerve are the two common NOOs. MRI is the investigation of choice in the diagnosis of schwannoma and the identification of NOO.

Keywords Schwannoma - Neurilemmoma . Nerve of origin · Extracranial $\cdot$ Head $\cdot$ Neck

\section{Introduction}

Extracranial head and neck schwannoma is a challenging condition to the head and neck surgeons. For tumours arising from the major cranial nerves, excision of tumour with the division of the nerve of origin (NOO) renders lifelong morbidity to the patients. On the other hand, other nerve-preserving excision method, e.g. intracapsular enucleation, does not guarantee intact nerve function after surgery. Because of the substantial chance of nerve palsy after operation, obtaining an accurate preoperative diagnosis, and preferably, with the identification of the NOO is crucial to the management of the disease. In this clinical review, a series of 30 cases of extracranial head and neck schwannoma will be analysed; symptomatology, usefulness of preoperative investigations, distribution of the NOO and the outcome of different tumour resection methods will be discussed.

\section{Methods}

Between January 2000 and December 2009, 30 patients with extracranial head and neck schwannoma were operated in our division. The medical records of these patients were reviewed. After history taking and physical examination, tissue diagnosis was pursued. For tumours which 
could be readily accessed on physical examination or through endoscopy, e.g. tumours in the oral and nasal cavities, incisional or punch biopsy was performed. Fine needle aspiration cytology (FNAC) was performed for tumours located at the superficial part of the neck. For tumours which were located at the deeper part of the neck, e.g. parapharyngeal space, it was usually not possible to obtain tissue for cytological or histological diagnosis before operation. In some patients, computed tomography (CT) or magnetic resonance imaging (MRI) was performed. In our division, both tumour excision methods, i.e. excision of tumour with the division of $\mathrm{NOO}$ and intracapsular enucleation of tumour, were practiced. The histological diagnosis and its clearance were confirmed on paraffin-embedded sections.

\section{Results}

\section{Symptomatology}

A total of 30 cases were analysed. The mean age at surgery was 41 years (range, 14-67 years). There were 16 male and 14 female patients. The mean size of tumour was $5 \mathrm{~cm}$ (range, $2-10 \mathrm{~cm})$. Among all 30 tumours, $23(77 \%)$ were located in the neck region, $2(7 \%)$ in the oral cavity, $2(7 \%)$ in the nasal cavity, $1(3 \%)$ in the skull base, $1(3 \%)$ in the middle ear and 1 $(3 \%)$ in the parotid region. Of the 23 tumours located in the neck region, 9 of which were located in the parapharyngeal space (Table 1). Most of the tumours $(18,60 \%)$ presented themselves as an asymptomatic palpable mass. Six (20\%) presented with nerve palsy (e.g. Horner's syndrome, vocal cord palsy); three (10\%) presented with pain and obstructive symptoms and three (10\%) were totally asymptomatic and were found incidentally on imaging studies.

\section{Preoperative investigations}

In our division, FNAC was performed for tumours located in the neck and parotid regions. FNAC would be repeated

Table 1 Anatomical position of 30 extracranial head and neck schwannomas

\begin{tabular}{ll}
\hline Neck & $23(77 \%)$ \\
Superficial neck & $14(47 \%)$ \\
Parapharyngeal space & $9(30 \%)$ \\
Oral cavity & $2(7 \%)$ \\
Nasal cavity & $2(7 \%)$ \\
Skull base & $1(3 \%)$ \\
Parotid gland & $1(3 \%)$ \\
Middle ear & $1(3 \%)$ \\
Total no. of tumour & $30(100 \%)$ \\
\hline
\end{tabular}

once if the result of the first FNAC was not informative. In this series, FNAC was performed in 20 cases, $10(50 \%)$ of whose results were inconclusive, $4(20 \%)$ showed the presence of spindle cells, 4 (20\%) suggested or confirmed the diagnosis of schwannoma and $2(10 \%)$ suggested a diagnosis other than schwannoma.

Contrast CT or MRI was performed in 26 cases. Sixteen $(62 \%)$ of these studies were not informative, which means that the studies were not able to suggest the diagnosis of schwannoma. Ten (38\%) suggested the diagnosis of schwannoma and the NOO could be pinpointed in one case.

\section{Surgery}

Most of the tumour $(20,67 \%)$ was resected through transcervical approach. Maxillary swing $(2,7 \%)$ and combined craniofacial approaches $(2,7 \%)$ were adopted for tumours located in the parapharyngeal space and skull base (Figs. 1, 2). In these 30 tumours, $22(73 \%)$ were resected with the division of the NOO, and $6(20 \%)$ were removed with intracapsular enucleation. There were two (7\%) cases of debulk operation with the preservation of NOO. Therefore, the NOO was preserved in eight cases. Nerve grafting was performed in two cases of facial nerve schwannoma in which the NOO was divided during operation.

\section{Nerve of origin}

The NOO was mainly determined by the postoperative neurological deficit and suggested by the intraoperative findings. The concordance rate of the identification of the NOO from intraoperative findings and postoperative neurological deficit was $87 \%$, which means that the NOOs determined by the intraoperative findings was the same as those determined by the postoperative neurological deficit

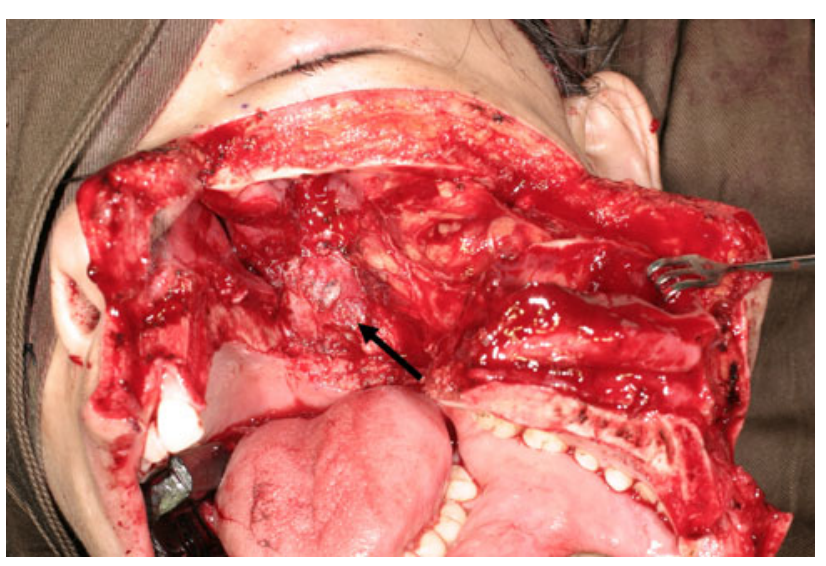

Fig. 1 A 41-year-old lady with trigeminal nerve schwannoma presented with nasal obstruction. The tumour (arrow) was resected through maxillary swing approach 


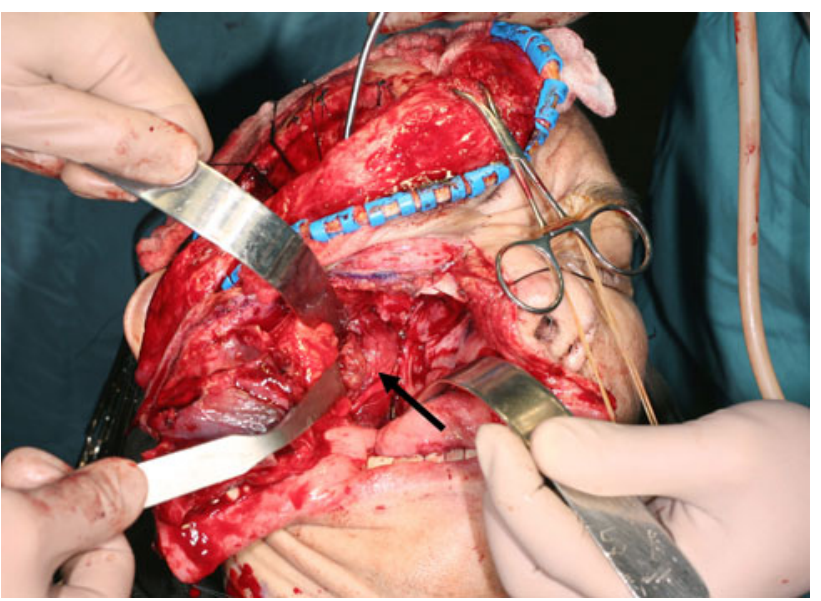

Fig. 2 A 57-year-old man with trigeminal schwannoma at the skull base. The tumour (arrow) was resected through combined craniofacial approach

in 26 cases. The distribution of the 30 NOOs was 10 (33\%) sympathetic trunk, 6 (20\%) vagus nerve, $3(10 \%)$ facial nerve. In five (17\%) cases, the NOO was not found to be arising from any major nerve (Table 2).

\section{Long-term outcome}

The mean follow-up period was 40 months (range, 1-119 months). There was no local recurrence in patients who had tumour resection with the division of NOO or intracapsular enucleation. In one patient who had debulk operation, follow-up MRI showed slow progression of the tumour.

For the six patients who had nerve palsies on presentation, their neurological deficits remained after operation and never recovered during the follow-up period. After excluding the five tumours which were not arising from major nerves and the two cases in which nerve grafting were performed, 23 cases were included in the analysis of neurological outcomes after operation. For these 23 cases, $15(65 \%)$ had the NOO divided, $6(26 \%)$ were intracapsular

Table 2 The nerve of origin (NOO) of 30 extracranial head and neck schwannomas

\begin{tabular}{ll}
\hline Sympathetic trunk & $10(34 \%)$ \\
Vagus nerve & $6(20 \%)$ \\
Facial nerve & $3(10 \%)$ \\
Trigeminal nerve & $2(7 \%)$ \\
Hypoglossal nerve & $1(3 \%)$ \\
Cervical plexus & $1(3 \%)$ \\
Brachial plexus & $1(3 \%)$ \\
Recurrent laryngeal nerve & $1(3 \%)$ \\
Minor nerve/NOO could not be identified & $5(17)$ \\
Total no. of schwannomas & $30(100 \%)$ \\
\hline
\end{tabular}

enucleation and 2 (9\%) were debulk operation. The NOO was therefore preserved in eight cases. The rates of nerve palsy after operation were $100 \%$ ( 15 cases), $67 \%$ (4 cases) and $50 \%$ ( 1 case) for resection with division of NOO, intracapsular enucleation and debulk operation, respectively.

\section{Discussion}

Schwannoma is a type of benign nerve sheath tumour. It arises from the Schwann cells which produce myelin sheath covering the cranial, peripheral and autonomic nerves. Therefore, schwannoma is always situated intimately with the NOO (Fig. 3). Extracranial head and neck schwannomas typically present as an asymptomatic neck mass. Obstructive symptoms may occur if the tumour gains enough size to compress on the upper aerodigestive tract. The pressure effect on the NOO can also cause nerve dysfunction. If a major nerve is involved, nerve palsy, e.g. vocal cord palsy, Horner's syndrome, sensory or motor dysfunction of the upper limb may result.

Both the incidence of malignant schwannoma and the rate of malignant transformation from benign schwannoma are not available in the literature. A review on intracranial malignant peripheral nerve sheath tumour (MPNST) suggested that most of the MPNSTs are developed sporadically rather than transforming from benign tumours [1]. Current knowledge also showed that most MPNSTs are associated with neurofibromatosis type $1[2,3]$. From these circumferential evidences, the rate of malignant transformation can be considered low in patients with solitary extracranial head and neck schwannoma. Because of the indolent nature and the remote chance of malignant

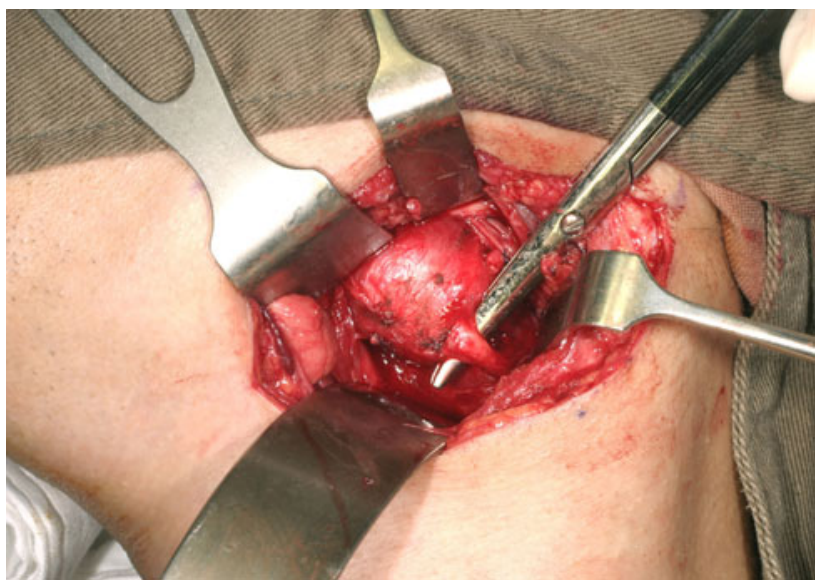

Fig. 3 47-year-old man with vagal schwannoma. The tumour is closely related to the nerve of origin. Intracapsular dissection is usually attempted for tumour arising from major nerves; however, an intact nerve function after operation is not guaranteed 
Fig. 4 Left the tumour is heterogeneously hyperintense on T2-weighted images. The heterogeneity of intensity may be explained by the tumour content. Right histology of the specimen showed spindle cell tumour with areas of haemorrhage, fibrosis and collagenous tissue encrusted with iron and calcium
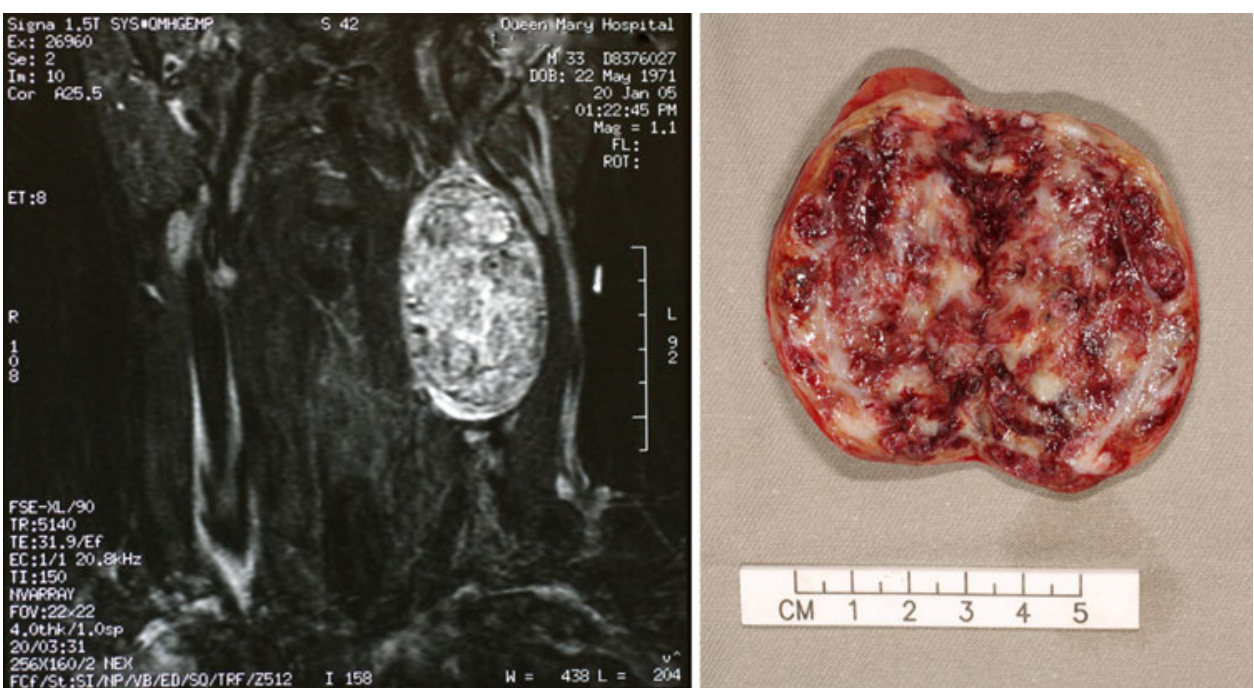

transformation, an observational approach is often possible. The decision of operation should, therefore, be based on the balance between the risk and benefit of the surgery, i.e. the severity of preoperative symptomatology and the anticipated postoperative neurological deficit.

The operation of schwannoma arising from major nerve can render lifelong morbidity to the patients. An accurate preoperative diagnosis with the identification of the NOO, therefore, allows patients to make an informed decision on whether to undergo operation or observation. The diagnosis of schwannoma is suggested by the clinical features, e.g. long-standing history, firm mass which is more mobile along the plane perpendicular to the course of the nerve and nerve palsy. FNAC and imaging studies are often ordered for diagnosis and planning of operation. However, in our series, the accuracy of FNAC was only $20 \%$. This figure echoes the findings from other studies that FNAC is neither sensitive nor specific in the diagnosis of schwannoma [4, 5]. The specificity of imaging studies (i.e. CT or MRI) was $38 \%$. The use of CT for diagnosis is not supported by the literature as there is no specific CT feature of schwannoma. Moreover, schwannoma may not be easily distinguished from paraganglioma in CT [6]. Currently, MRI has superseded CT because of better soft tissue delineation. Studies on MRI also found the NOO can be determined from the spatial relationship between the tumour and the great vessels of the neck [7, 8]. For the MRI findings in our series, the tumours were hypointense on T1-weighted images and heterogeneously hyperintense on T2-weighted images. The heterogeneity of intensity may be due to a mixture of haemorrhage, fibrosis and calcium deposits inside the tumour (Fig. 4). The NOO could be visualized in some cases with MRI but none with CT (Fig. 5).

The choice of operation, i.e. excision with the division of NOO or intracapsular enucleation and its success in

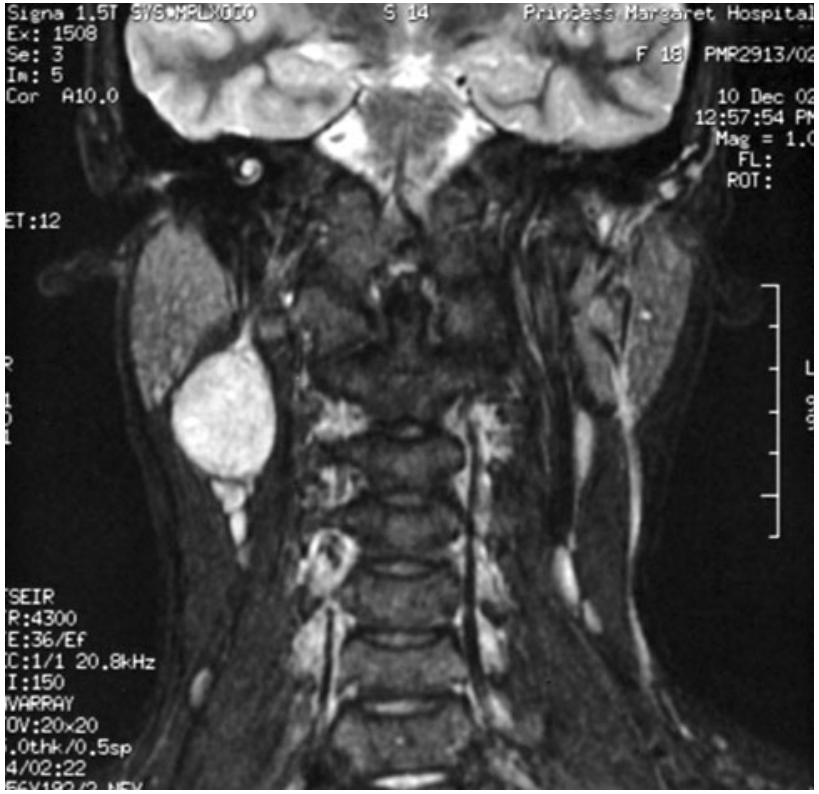

Fig. 5 MRI showed vagal schwannoma and the vagus nerve. The relationship between the schwannoma and its nerve of origin can be better appreciated with MRI than CT

preserving nerve function are mainly determined by the relationship between the tumour and the NOO. Intracapsular enucleation is most feasible for tumour which is situated eccentrically without being splayed by nerve fibres. It is usually attempted for schwannomas arising from major nerves, e.g. vagus nerve, brachial plexus. The recurrence rate after intracapsular dissection is not well documented in the literature. According to two recent small-scale series on schwannoma, no recurrence was found after intracapsular dissection within their study periods $[9,10]$. In this series, we also did not detect any recurrence after intracapsular dissection during the follow-up. 
The treatment of postoperative nerve palsy should be regarded as a part of the management of schwannoma. As the sympathetic trunk and the vagus nerve are the common NOOs, corrective procedures, e.g. levator operation and medialization of vocal cord should be explained to the patients before operation. In our series, in patients who presented with nerve palsy, their neurological deficits remained even after operation and never recovered during the follow-up period. Corrective procedures can therefore be offered on the same setting of the tumour resection surgery.

\section{Conclusion}

The management of extracranial head and neck schwannoma is an art. An accurate preoperative diagnosis of schwannoma with the identification of the NOO allows the patients to make an informed decision on whether to undergo operation or observation. At present, MRI appears to be the investigation of choice for diagnosis and identification of NOO.

\section{Conflict of interest None}

Open Access This article is distributed under the terms of the Creative Commons Attribution Noncommercial License which permits any noncommercial use, distribution, and reproduction in any medium, provided the original author(s) and source are credited.

\section{References}

1. Ziadi A, Saliba I (2010) Malignant peripheral nerve sheath tumour of intracranial nerves: a case series review. Auris Nasus Larynx 37:539-545
2. Tucker T, Wolkenstein P, Revuz J, Zeller J, Friedman JM (2005) Association between benign and malignant peripheral nerve sheath tumors in NF1. Neurology 65:205-211

3. Pasmant E, Masliah-Planchon J, Lévy P, Laurendeau I, Ortonne N, Parfait B, Valeyrie-Allanore L, Leroy K, Wolkenstein P, Vidaud M, Vidaud D, Bièche I (2010) Identification of genes potentially involved in the increased risk of malignancy in NF1microdeleted patients. Mol Med [Epub ahead of print]

4. Yu GH, Sack MJ, Baloch Z, Gupta PK (1999) Difficulties in the fine needle aspiration (FNA) diagnosis of schwannoma. Cytopathology 10:186-194

5. Biswas D, Marnane CN, Mal R, Baldwin D (2007) Extracranial head and neck schwannomas-a 10-year review. Auris Nasus Larynx 34:353-359

6. Zhang H, Cai C, Wang S, Liu H, Ye Y, Chen X (2007) Extracranial head and neck schwannomas: a clinical analysis of 33 patients. Laryngoscope 117:278-281

7. Miller FR, Wanamaker JR, Lavertu P, Wood BG (1996) Magnetic resonance imaging and the management of parapharyngeal space tumors. Head Neck 18:67-77

8. Saito DM, Glastonbury CM, El-Sayed IH, Eisele DW (2007) Parapharyngeal space schwannomas: preoperative imaging determination of the nerve of origin. Arch Otolaryngol Head Neck Surg 133:662-667

9. Ozdemir O, Ozsoy MH, Kurt C, Coskunol E, Calli I (2005) Schwannomas of the hand and wrist: long-term results and review of the literature. J Orthop Surg (Hong Kong) 13:267-272

10. Kim SH, Kim NH, Kim KR, Lee JH, Choi HS (2010) Schwannoma in head and neck: preoperative imaging study and intracapsular enucleation for functional nerve preservation. Yonsei Med J 51:938-942 